\title{
Strength Durability-Based Design Mix of Self-Compacting Concrete with Cementitious Blend using Hybrid Neural Network-Genetic Algorithm
}

\author{
John Pepard Mendoza Rinchon ${ }^{1 *}$ \\ ${ }^{I}$ Faculty Member, Department of Civil Engineering, College of Engineering and Architecture \\ Technological Institute of the Philippines, Quezon City, Philippines 1109 \\ *Corresponding author: jprinchon27@gmail.com
}

\begin{abstract}
Sustainable development in self-compacting concrete (SCC) has been studied extensively for the recent years for the purpose to address its growing demand in construction projects. Sustainable SCC can be defined as concrete mix with partially replaced cement content that varies from low to high level using different mineral admixtures. Silica fume and fly ash which is considered as the most common sustainable mineral admixtures for binary and ternary cementitious blends show good effect to the compressive strength and chloride penetration resistivity of hardened SCC. In this paper, this effect was further investigated by using two widely used biological inspired computing models namely the artificial neural network (ANN) and genetic algorithm (GA). The test results of compressive strength and chloride ion penetration resistance from thirty-six concrete samples with varying replacement level of binary and ternary cementitious blends were utilized as inputs for model development. ANN was used to obtain models that describe analytically the relationship of material components to the compressive strength and chloride penetration resistivity. The derived models were further explored through optimization using GA. Results shows that ANN was able to establish the relationship of strengthdurability parameters to the material components while GA is able to derived optimal mix proportion for best strengthdurability performance. The present study also validates the sensitivity of the replacement level of silica fume and fly ash as a ternary cementitious blend to the strength-durability performance of SCC. This indicates that high volume content of ternary blended cement can improve chloride penetration resistivity and exhibited high compressive strength.
\end{abstract}

Keywords-Artificial Neural Network, Genetic Algorithm, Self-compacting Concrete, Sustainable Concrete

\section{INTRODUCTION}

Sustainable development in self-compacting concrete (SCC) has been introduced and studied extensively over the last few years. This is to address the unattractive perception of negative environmental impact associated in the growing demand of SCC technology in construction projects; an increase in usage of Portland cement results to high carbon footprint value. Therefore, reduction of cement was introduced to develop greener SCC [1]. One way to reduce the use of Portland cement in SCC is the use of mineral admixtures such as silica fume, fly ash, ground granulated blast furnace slag, and other cementitious materials as partial replacement. This reduction is also economical especially if the mineral admixtures are industrial by-products or wastes [2].

Findings of different literatures reveals the good effect of different mineral admixtures to the properties of fresh and hardened SCC. Mohamed and Al-Hawat [3] reported the improvement of SCC's resistance to chloride ion penetration using low volume replacement $(\leq 40 \%)$ of binary blend fly ash and basalt fibers. Silica fume and fly ash under high volume replacement $(\geq 40 \%)$ also improved this durability parameter and indicated that ternary blend of these minerals is more effective compared to binary blend to the early strength of concrete [4]. High level cement replacement using single, binary, and ternary blends of fly ash, silica fume, and ground granulated blast furnace slag also exhibited good performance in saturated water absorption test [5]. Mineral admixtures like fly ash and limestone powder are reported good in reducing the dosage of superplasticizer necessary to obtain desired slump. Theses minerals improve the rheological properties and reduce the risk of cracking due to heat of hydration [610]. Gesoğlu et al. [11] also investigated the performance of SCC containing binary, ternary, and quaternary cementitious blends of fly ash, blast furnace slag, and silica fume in terms of rheology, strength, and durability requirements. Moreover, multi-objective optimization using desirability function technique was performed to achieve optimal concrete mix proportions.

Cement replacement using different mineral admixtures has proven to be viable solutions to develop sustainable SCC under desired workability, durability, and strength. Concha et al. [12, 13] explored further the established influences of two types of mineral admixtures to the workability parameters of SCC. This is done through the aid of Artificial Neural Network (ANN) and Genetic Algorithm (GA) as prediction model and optimization technique respectively. ANN established the constitutive relationship of mineral admixtures along with SCC components to slump flow, L-box ratio, and screen stability ratio. Furthermore, GA derived optimal concrete mix proportion with high rheological performance.

Computing models like ANN and GA belong to large class of artificial intelligence inspired by biological and natural processes intended to solve specific problem. ANN is a type of machine learning algorithm based on human brain mechanism and is commonly used as prediction model. Figure 1 (a) shows the typical ANN architecture. This model network is basically developed using existing training data set containing pairs of inputoutput elements needed to train the system of interconnected neurons [14]. GA on the other hand is an iterative algorithm based on genetics and natural selection ruled by Darwinian theory of evolution "survival of the fittest". This is commonly deployed as optimization and search technique $[15,16]$. The flow chart of GA is shown in Figure 1 (b). Solution of optimization problem in GA is represented by a 
The Third International Conference on Civil Engineering Research (ICCER)

August $1^{\text {st }}-2^{\text {nd }}$ 2017, Surabaya - Indonesia

chromosome while population is a group of chromosomes or possible solutions. Each chromosome is composed of genes that represent decision variables. This gene can be represented either numerical, binary, or hard coded symbols depending on the problem considered. In addition, initial generation of randomly selected chromosome must undergo genetic operators such as fitness evaluation, crossover, mutation, and selection in order to produce new and better generation. This process is repeated until generation of a chromosome or population with satisfactory fitness values meet. Thus, this final generation of chromosomes are considered to be the optimal solution or set of solutions.

The present paper generally aims to utilize ANN and GA to develop a hybrid model for prediction and optimization purposes. ANN was used to establish separate models that would describe the relationship of strength and durability parameters to the components of SCC containing ternary cementitious blend of silica fume and fly ash. These models were optimized using GA to derive the optimal mixture proportion of SCC with high strength-durability performance. Detailed discussion of model development and hybrid algorithm in this study are presented in the first part while results and discussion as well as conclusions are presented in the last part.

\section{METHOD}

\section{A. Materials}

The study utilized the data and results published by Wongkeo et al. [4] as primary datasets. Consequently, thirty-six datasets of self-compacting concrete (SCC) samples with corresponding test results of 28-day compressive strength and chloride ion penetration resistance were retrieved and summarized (See Figures 2 and 3). These datasets were used as inputs for hybrid model development. The concrete sample contains blended cement of silica fume and fly ash at high volume replacement level of 50, 60, and 70\%. Each material component of SCC was prepared in accordance with the guidelines and testing procedures set by American Society for Testing of Materials (ASTM). Mix proportions of SCC are shown in Table 1. Moreover, the proportioning of material component of SCC was based on the following assumptions: fixed water content at 180 $\mathrm{kg} / \mathrm{m}^{3}$; water to binder $(\mathrm{w} / \mathrm{b})$ ratio of $0.3,0.35$, and 0.4 were used; fixed coarse aggregate to total aggregate ratio at 0.35 ; fine aggregate to total aggregate in range of 0.65 ; and adjusted superplasticizer dosage for constant slump flow of $600 \pm 50 \mathrm{~mm}$.

For concrete sample preparation and testing, a 100 mm-cube concrete specimen was used for compressive strength test in accordance with British Standards [17]. ASTM: C102 was adopted for chloride ion penetration resistance test for a $100-\mathrm{mm}$ diameter by $200-\mathrm{mm}$ high cylindrical concrete specimen.

\section{B. Model Development}

This section is divided into two subsections to discuss the development of hybrid model. Subsection (1) discussed the assumptions and methodology in constructing the architecture of predicting models using artificial neural network. Meanwhile, the full discussion of the implementation of genetic algorithm is presented in subsection (2). Furthermore, both computing models were deployed in licensed version of MatLab® R2015a program.

\section{(1) Artificial Neural Network (ANN)}

Feedforward multilayered supervised neural network with error back-propagation algorithm was selected for this study from the large class of ANN models. It was use because of its simplicity and for being widely used ANN model. The architecture of ANN consists of artificial neurons analogous to natural neurons of the human brain that are clumped into series of input, hidden and output layers. According to Chopra et al. [18] there are three essentials to consider before structuring the architecture of ANN model, namely: 1) Topology organization and interconnection of a neural network into layers; 2) Learning - information storage in the network; and 3) Recall - retrieval of information from the network. These essentials are reflected with the following internal parameters: 1) performance function, 2) learning function, 3) weights and biases, 4) hidden layers and neurons, 5) and transfer function.
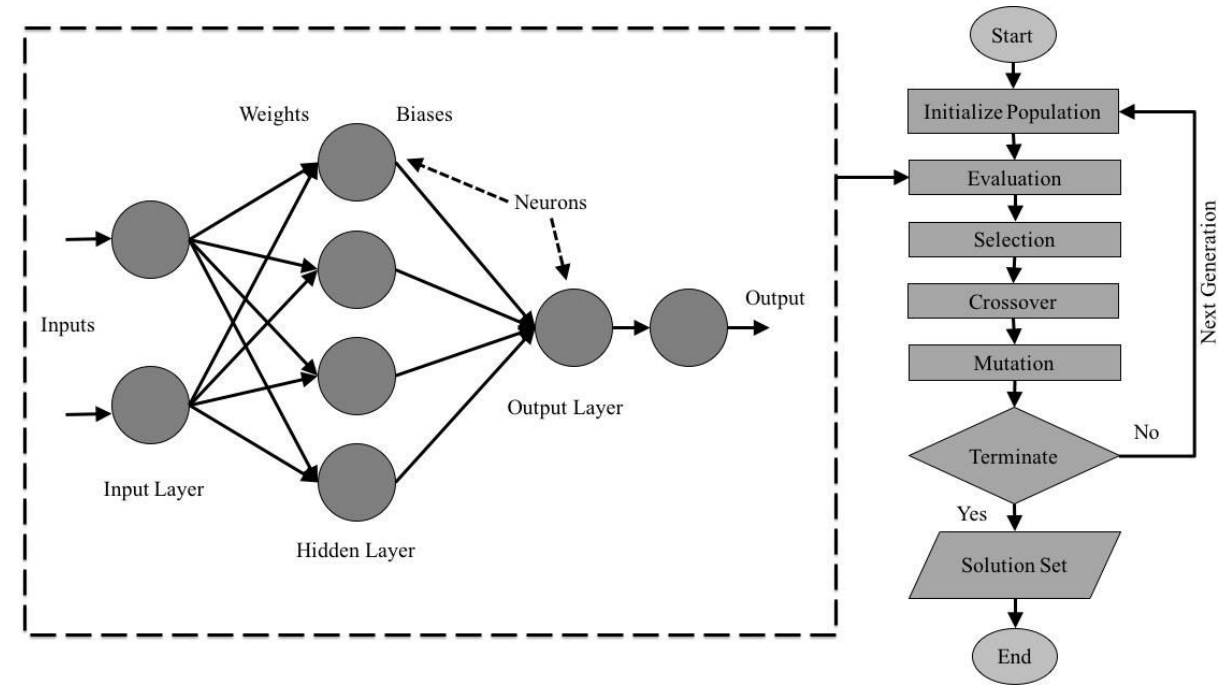
Table 1. Summary of mix proportions of SCC and experimental results by Wongkeo et al. [4]

Figure 1. Hybrid model (a) Artificial neural network architecture (b) Genetic algorithm flow chart

\begin{tabular}{|c|c|c|c|c|}
\hline \multirow{2}{*}{ Variable } & \multicolumn{4}{|c|}{ Details of Variable } \\
\hline & Minimum & Maximum & Range & Mean \\
\hline \multicolumn{5}{|l|}{ Input } \\
\hline Portland Cement, $P C\left(\mathrm{~kg} / \mathrm{m}^{3}\right)$ & 135 & 600 & 465 & 280.3 \\
\hline Fly Ash, $F A\left(\mathrm{~kg} / \mathrm{m}^{3}\right)$ & 180 & 420 & 240 & 286.9 \\
\hline Silica Fume, $S F\left(\mathrm{~kg} / \mathrm{m}^{3}\right)$ & 23 & 60 & 37 & 39.2 \\
\hline Fine Aggregates, $F\left(\mathrm{~kg} / \mathrm{m}^{3}\right)$ & 908 & 1,166 & 258 & $1,025.9$ \\
\hline Coarse Aggregates, $C A\left(\mathrm{~kg} / \mathrm{m}^{3}\right)$ & 595 & 640 & 45 & 618.7 \\
\hline Superplasticizers, $S P\left(\mathrm{~kg} / \mathrm{m}^{3}\right)$ & 0.59 & 9.45 & 8.87 & 3.4 \\
\hline \multicolumn{5}{|l|}{ Output } \\
\hline 28-Day Compressive Strength, f'c (MPa) & 28.2 & 100.5 & 72.3 & 62.0 \\
\hline Total Charge Passed, $Q$ (Coulomb) & 266 & 4,659 & 4,393 & $1,636.6$ \\
\hline
\end{tabular}

Table 2. Artificial neural network model internal parameters

\begin{tabular}{ll}
\hline Parameter & Value \\
\hline Training algorithm & Levenberg-Marquardt Algorithm \\
Transfer function & Hyperbolic Tangent Sigmoid (Tansig) \\
Performance function & Mean Square Error (MSE), Pearson Correlation Coefficient (R) \\
Number of hidden layers & $1 ; 2$ \\
Number of neurons per hidden layer & $(\text { nip }-1)^{*}$ to 20 \\
Performance Goal & 0.000001 \\
Epochs & 10,000 \\
\hline
\end{tabular}

\section{(a) ANN internal parameters}

Good concept of the impact of internal parameters (See Table 2) will dictate the successful application of ANN in establishing the relationship of input and output elements. Furthermore, iterative experimental exploration through trial and error of theses internal parameters was conducted in order to arrive at the best possible parameters capable of prediction.

The present study deployed hyperbolic tangent sigmoid function or tansig function and Levernberg-Marquardt algorithm as transfer and training functions respectively. According to Asteris et al. [19] no prior reasons as to why tansig function should always provide optimal decision boarders although the choice of the transfer function has always have strong influence on the complexity and performance of the neural network. Transfer functions commonly serve as activation functions for the connection between weights of a neuron and input element. Specifically, tansig function operates by returning outputs compressed between -1 and 1 in which it has an ability to learn complex nonlinear relation between the input and output parameters. On the other hand, Levernberg-Marquardt learning function is the most suitable algorithm for concrete related data according to available literatures [18, 19]. It is attributed to be significantly high speed training method especially for moderately sized feedforward neural networks as well as non-linear problems. This function is also readily deployable in MatLab® program.

According to Taner [20], more hidden layers can be used to handle complicated and erratic cases. However, Zhang et al. [21] suggested that most literatures opted to use one or two hidden layers for modelling. In this present work, the number of neurons in hidden layer will be part of the experimental exploration. While the performance of ANN models was assessed using mean square error (MSE) and Pearson correlation coefficient (R), high performing ANN model has MSE and R values equal to zero and one respectively. In addition, satisfactory values for R and MSE are used as stopping criteria for the multiple training simulation in deriving the final weights and biases for high performing ANN models.

Furthermore, ANN modeling is divided into three parts (i.e. training, validation, and testing). Training is the first part of the modeling in which formulation of the initially structured model is executed. Consequently, validation is used to derive final weights and biases while testing is to ensure the accuracy of the final derived model. In addition, the provided data sets are distributed to training, validation, and testing parts using 70, 15, and $15 \%$ proportion.

\section{(2) Genetic Algorithm (GA)}

The second stage of hybrid model is deployment of GA originally proposed by Goldberg [22] that is generally composed of three operators known as the selection, mutation and crossover. In addition, formulation of objective functions for strength and durability optimization problems is the initial step of this stage. The objective functions were expressed as follows:

Maximize: $f^{\prime} c=\mathrm{f}(P C, F A, S F, F, C A, S P)$

Minimize: $Q=\mathrm{f}(P C, F A, S F, F, C A, S P)$

where $f^{\prime} c$ - 28-day compressive strength of SCC (MPa); $Q$ - charge passed (Coulombs); $P C$ - Portland cement $\left(\mathrm{kg} / \mathrm{m}^{3}\right) ; F A-$ fly ash $\left(\mathrm{kg} / \mathrm{m}^{3}\right) ; S F-$ silica fume $\left(\mathrm{kg} / \mathrm{m}^{3}\right) ;$ $F$ - fine aggregates $\left(\mathrm{kg} / \mathrm{m}^{3}\right) ; C A$ - coarse aggregates 
$\left(\mathrm{kg} / \mathrm{m}^{3}\right) ; S P$ - superplasticizer $\left(\mathrm{kg} / \mathrm{m}^{3}\right) . f^{\prime} c$ and $Q$ are the predicting models developed through ANN modeling which serve as functions that establish the relationship between material components of SCC and strengthdurability parameters.

Figure 2. 28-day compressive strength of different SCC mix proportions with cementitious blends at w/b ratio of $0.3,0.35$, and 0.4

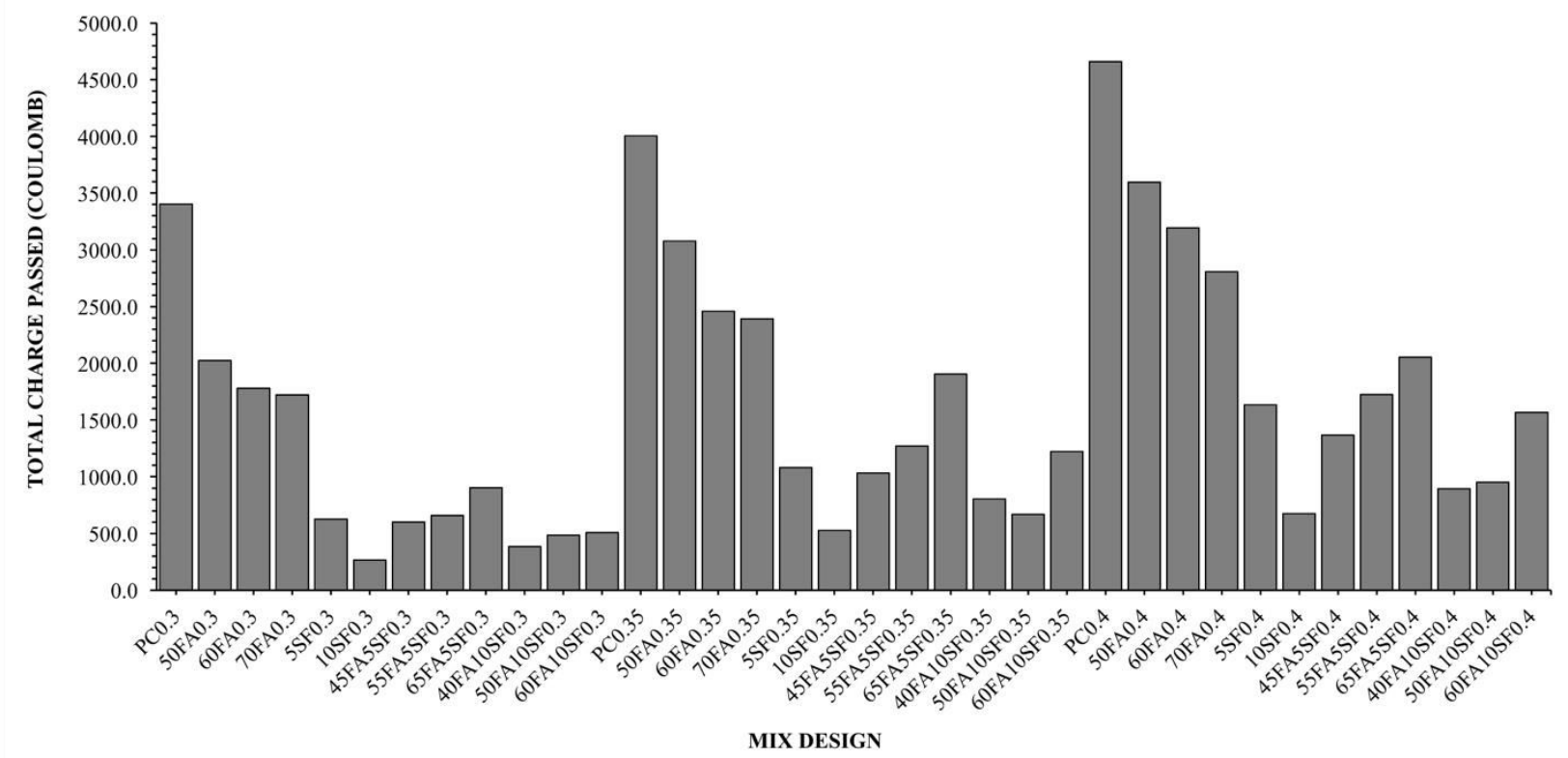

Figure 3. Chloride resistance (total charge passed) of different SCC mix proportions with cementitious blends 


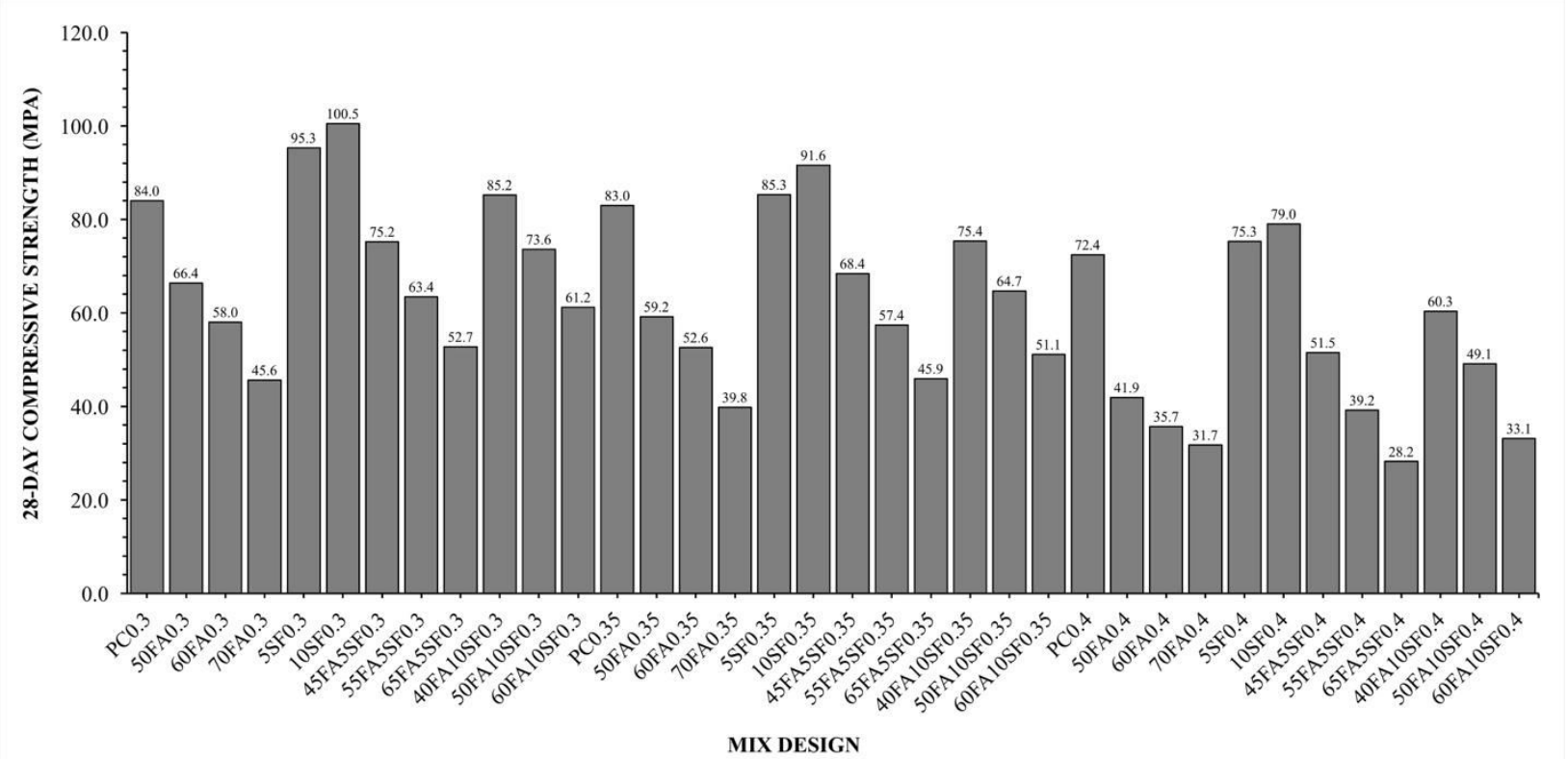

In this research, three GA models were developed to find the optimal mix proportion of SCC under high strength and durability performance. CSNN1 and QPNN1 are the objective functions based on $f^{\prime} c$ and $Q$ ANN models respectively. These were used in separate single-objective GA optimization. CSNN1 was used to determine SCC mix proportion with high performing 28day compressive strength while QPNN1 was used for solving optimal mix proportion that exhibited high chloride ion penetration resistance (low charge passed in coulomb). The third model was a multi-objective GA optimization in which CNN1 was used as the objective function for determining the pareto-front of optimal SCC mix proportions. This CNN1 model is a single code generated ANN model of 28-day compressive strength and charge passed for chloride ion penetration resistance.

In the deployment of GA, proper assignment of available method for each three fundamental GA operators (i.e. selection, mutation, and crossover) is vital.
This will influence the convergence rate and capability of GA models in search for global solutions of the optimization problems. Full discussion of each operator were presented as follows:

\section{(a) Selection}

Selection is a GA operator essentially based on theory of Darwinian natural selection. This is the first operator in the whole GA procedure wherein initial chromosomes or possible solutions were selected. Proper method and stress of selection operator provide the driving force of GA search. This is critical in directing the algorithm toward promising and wider regions of the search space. According to Lim et al. [23], commonly at the start of the genetic algorithm search, low selection stress was specified in favor of a wide exploration of the search space while, at the latter part, high selection stress was recommended in order to achieve the most promising regions in the search space. 
In the present research, the stochastic uniform and tournament methods were utilized as selection operators. Stochastic uniform or stochastic universal sampling (SUS) selection developed by Baker [24] was used in the

\section{(b) Mutation}

Mutation operator is a special feature of genetic algorithm because of its mechanism that preserves specific characteristic of the initial chromosomes needed

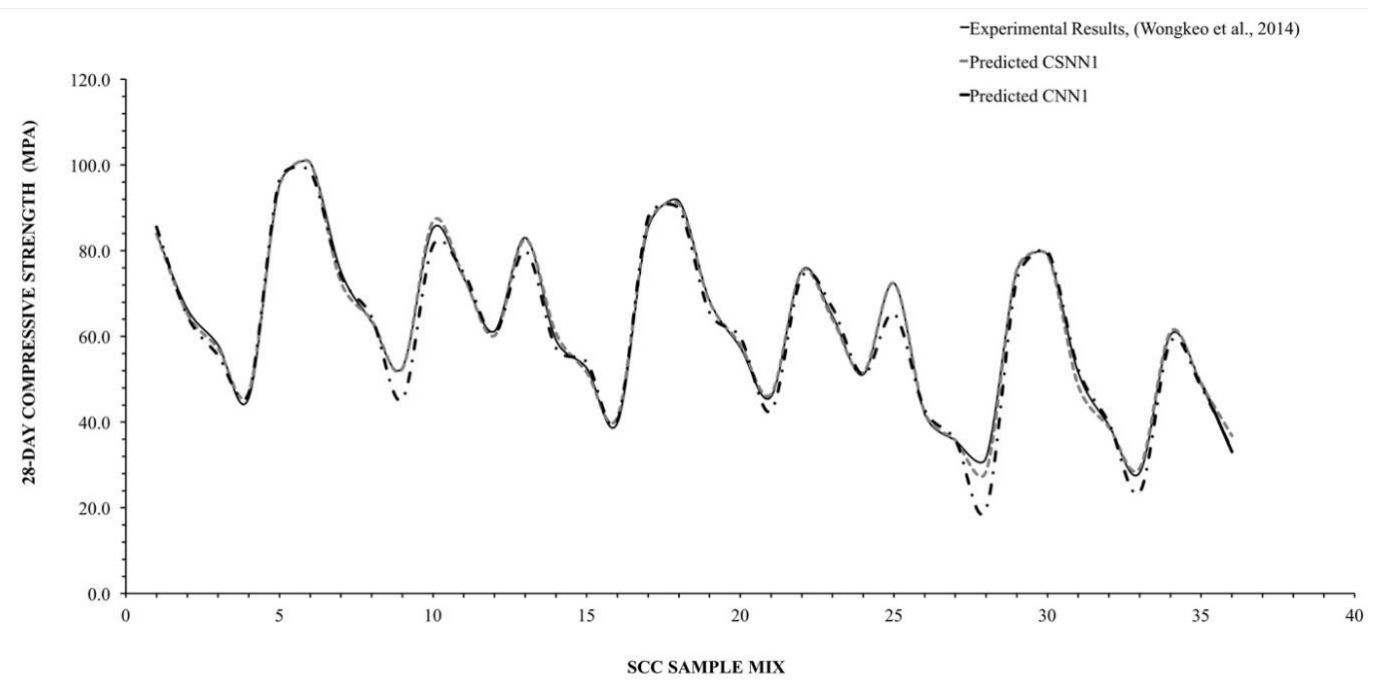

Figure 4. Comparison among experimental results, CSNN1 and CNN1 prediction of 28-day compressive strength

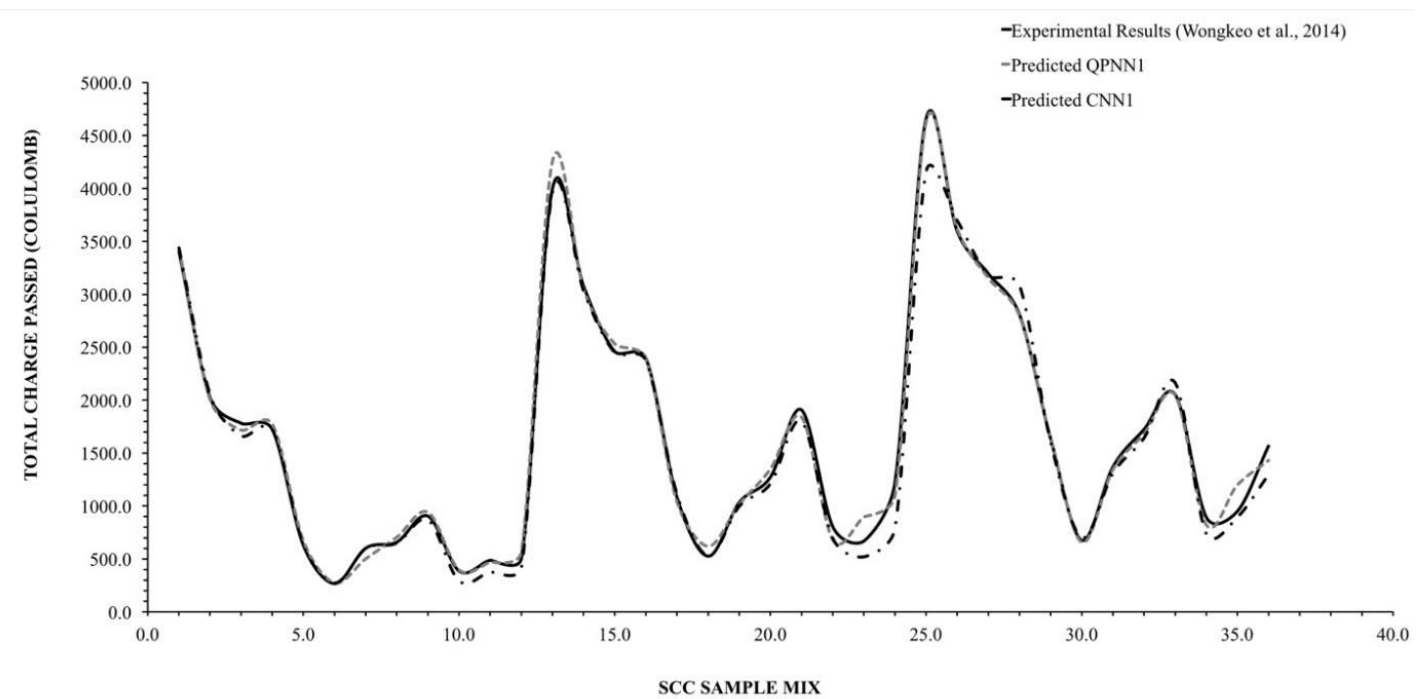

Figure 5. Comparison among experimental results, QPNN1 and CNN1 prediction of charge passed for chloride ion penetration resistance

single objective optimization problems. This is because SUS is one of the most popular selection methods particularly because of its capability in achieving minimum spread of generated population and zero bias selection of individuals. Rank and top fitness scaling methods were combined to SUS to enhance the selection operator of the whole GA process. On the other hand, binary tournament was used in multi-objective optimization problem. Generally, tournament selection randomly chooses two-chromosome set and pick out high performing one from the set and proceed to reproduction. These chromosomes are inserted into new population and the process is repeated until the population becomes full. in locating promising solutions. This feature compensates the negative effect of crossover operator of losing specific characteristic during the generation of new population. The mutation process is performed by using mutation rate that is defined as percentages of introducing new genes over total number of genes in the population needed for trial. Very slow mutation rate will yield to local solution due to unexplored useful characteristics of other possible chromosomes. However, high rate will cause random perturbation of the new population loosing resemblance to its parent population and later the algorithm loses its track from learning the record of the search. To avoid this, adaptive feasible mutation was used for single and multi-objective optimization problems

[25]. 


\section{(c) Crossover}

Crossover operator generally operates under the mechanism of sexual generation of a new chromosome from two parent chromosomes. The mechanism takes part of the total characteristics of one parent chromosome and other parts from the other parent chromosome and combining both to generate to new chromosome. The process operated and controlled by crossover rate that defined the number of new chromosomes in each generation to the population size that will undergo the operation. A higher crossover rate allows the algorithm to explore the large region of the solution space and reduces the probability of landing for a local optimum but if this rate is too high, this results to computationally intense in exploring unpromising regions of the solution space [23]. Scattered, one-point, two-point, and intermediate crossover were utilized for both single objective and multi-objective optimization problems. These crossover models are all deployable in MatLab® program.

\section{(d) Constraints}

In order to attain global solution and realistic values for the optimization problems, the following constraints and bounds were formulated as follows:

$$
\begin{aligned}
& 135 \leq P C \leq 600 \\
& 180 \leq F A \leq 420 \\
& 23 \leq S F \leq 60 \\
& 908 \leq F \leq 1,166 \\
& 595 \leq C A \leq 640 \\
& 0.59 \leq S P \leq 9.45 \\
& 0.3 \leq 180 /(P C+F A+S F) \leq 0.4 \\
& 0.6 \leq F /(F+C A) \leq 0.65 \\
& C A /(F+C A)=0.35
\end{aligned}
$$

Equations three to eight were implemented for single objective GA optimization of both CSNN1 and QPNN1 models. Equation nine to 11 were additional constraints for multi-objective GA optimization of CNN1 model in solving the strength-durability problem.

\section{RESULTS AND DISCUSSION}

\section{A. Compressive strength ANN model}

After several experimental exploration of the different significant internal parameters, final ANN model for the 28-day compressive strength was derived (i.e. CSNN1 model). Final internal parameters included six hidden neurons with two hidden layers. Tansig transfer function was still proven to be high performing in the input and output signal processing thus making the model capable to handle dimensionally varied input and output parameters. For training algorithm, LevenbergMarquardt algorithm is observed to be efficient at high rate convergence for moderately sized feedforward neural networks with non-linear problems. The performance of the CSNN1 model as shown in Figure 6 (a). Pearson correlation coefficient, R values for training, validation, and testing were all considerably high performing with values greater than 0.99. CSNN1 model has overall $\mathrm{R}$ value of 0.99774 . These $\mathrm{R}$ values remarked that the derived model has good predicting capability. Figure 4 presented the comparison of the experimental values of 28-day compressive strength to the predicted values by CSNN1 model using 36 datasets. It was clear that the experimental values and predicted values were very close.

\section{B. Charge passed ANN model}

QPNN1 as the final derived ANN model for the chloride ion penetration resistance is also remarked to be high performing in establishing the relationship of the SCC's material components to the total charge passed. Figure 5 presented the high performance of QPNN1 based on the comparison between the predicted and experimental values of 36 datasets simulation. The performance also reflected with the $\mathrm{R}$ values of training, validation, and testing sets which are all greater than 0.99 as presented in Figure 6 (b). The final architecture of QPNN1 is composed of five hidden neurons with two hidden layers. Levenberg-Marquardt algorithm and tansig function were also the final training and transfer functions respectively. The QPNN1 has overall $\mathrm{R}$ value of 0.99679 .

\section{Combined ANN model}

The third model is the combination of the two output parameters in a single ANN model. This is for the purpose of deriving the objective functions of the multiobjective GA optimization problem in a single ANN model. The final derived combined ANN model (i.e. CNN1) also underwent performance assessment and training processes. The predicted values of this model for the 28-day compressive strength and charge passed are also compared to the experimental results as presented in figures 4 and 5. The final architecture was composed of seven hidden neurons and two hidden layers under satisfactory R values of 0.99619 as presented in figure 6 (c). Tansig function and Levenberg-Marquardt algorithm were also the transfer and training functions of the final derived model.

$\mathrm{R}$ values for three models under training, validation, testing and overall stage were summarized in table 3 .

Table 3. Pearson correlation coefficient, R performance for three ANN models

\begin{tabular}{lllll}
\hline \multirow{2}{*}{ Neural Network Model Code } & \multicolumn{4}{l}{ Pearson correlation coefficient, R-Value } \\
\cline { 2 - 5 } & Training & Validation & Testing & All \\
\hline CSNN1 & 0.99951 & 0.99052 & 0.99645 & 0.99774 \\
QPNN1 & 0.99623 & 0.99963 & 0.99841 & 0.99679
\end{tabular}



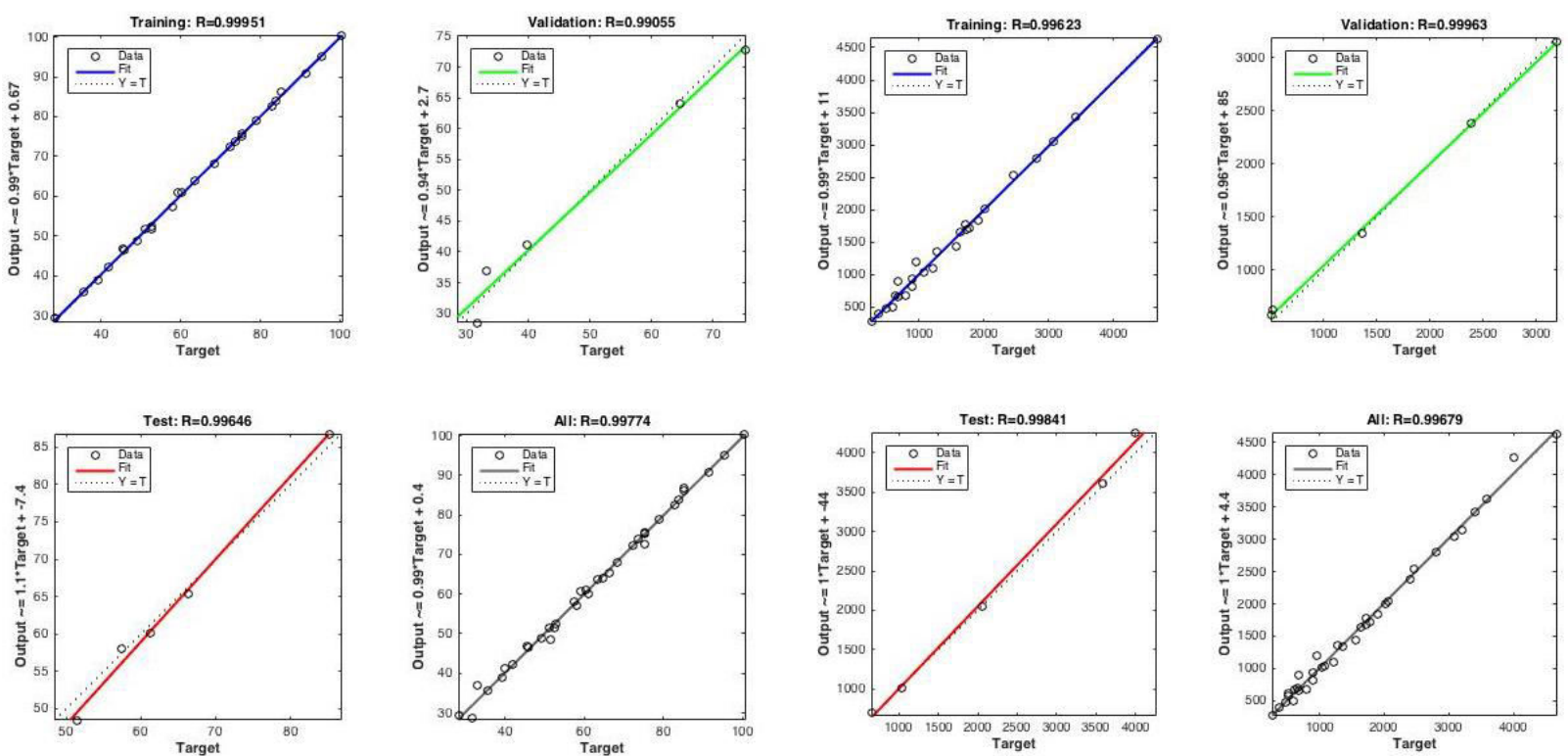

(a)
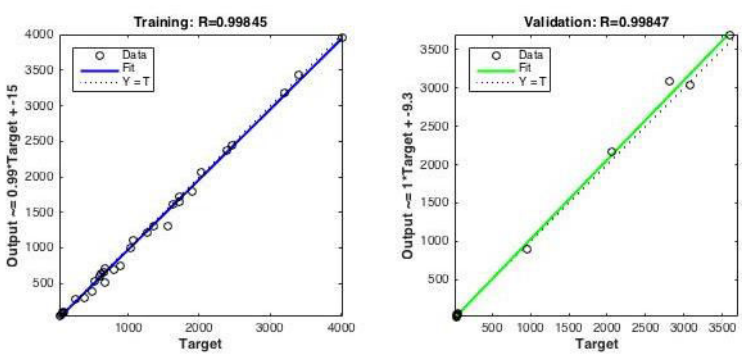

(b)
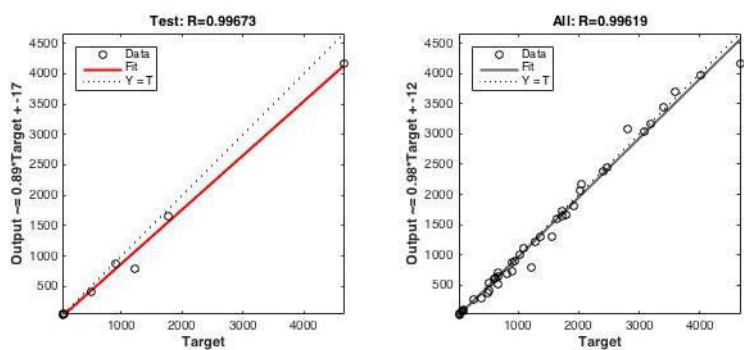

(c)

Figure 6. Pearson correlation coefficient, R values for (a) CSNN1 (b) QPNN1 and (c) CNN1

CNN1

0.99845

0.99846

0.99673

0.99619

D. Single objective GA optimization

In this stage, applicability of GA as stochastic search technique was tested. Two separate single-objective optimization problems were solved by GA. GA was able to determine the optimal solutions for the two problems that involved the strength and durability requirements of sustainable SCC containing cementitious blends.

Table 4 presents the two optimal mix proportions of sustainable SCC that correspond to individual objective performance. Mix no. 1 is the optimal proportion for high compressive strength SCC. The objective performance of this design mix $f^{\prime} c=114 \mathrm{MPa}$ in which it can be considered as a high strength concrete. It has FA and SF content of $39 \%$ and $5.6 \%$ of the total binder weight respectively. The second mix proportion performed with high chloride resistance at $<0$ charge passed (Negligible). FA content at $40 \%$ while SF content at $3.7 \%$ of the total binder weight. Both mix proportions (i.e. nos. 1 and 2 ) have a $w / b$ ratio $=0.17$, and the same were determined after running the GA over 300 generations as shown in figures 7 and 8 .

The lower w/b ratio for both proportions affirmed with previous literatures, that decreasing w/b ratio corresponds to increasing of compressive strength and chloride ion penetration resistance. Low compressive strength and chloride penetration resistance was accompanied by high porosity caused by increased $\mathrm{w} / \mathrm{b}$ ratio $[4,26]$.

It was also observed the good effect of FA and SF as ternary cementitious blend to the compressive strength and chloride ion penetration resistance of SCC. Because of the dynamic relationship between FA and SF. SF tends to compensate the low pozzolanic reaction of FA and serves as micro filler. Moreover, FA and SF improve 
both the physical and chemical chloride binding effect

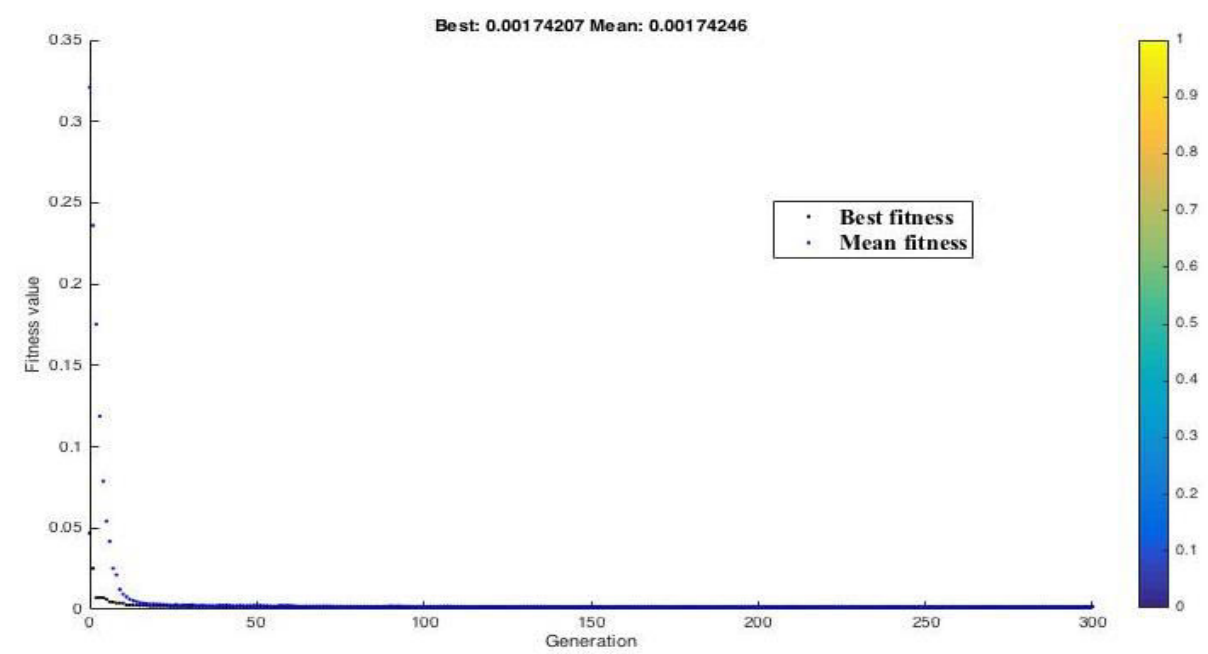

Figure 7. Results of genetic algorithm generation for $f^{\prime} c$ optimization problem (CSNN1)

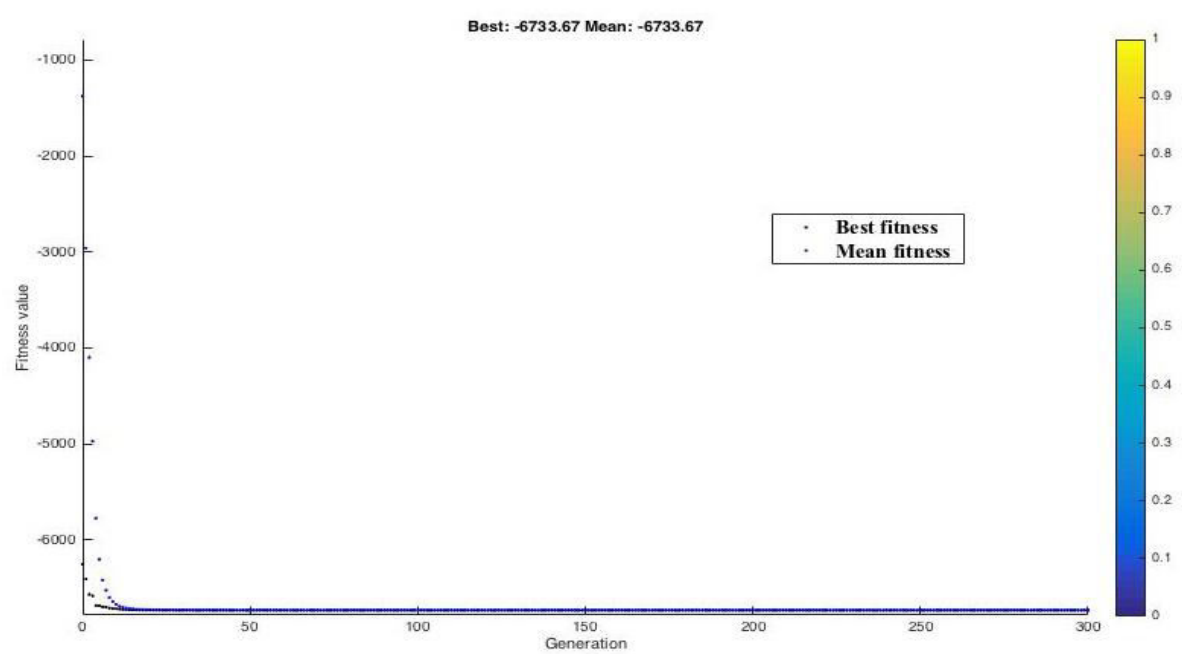

Figure 8. Results of genetic algorithm generation for $Q$ optimization problem (QPNN1)
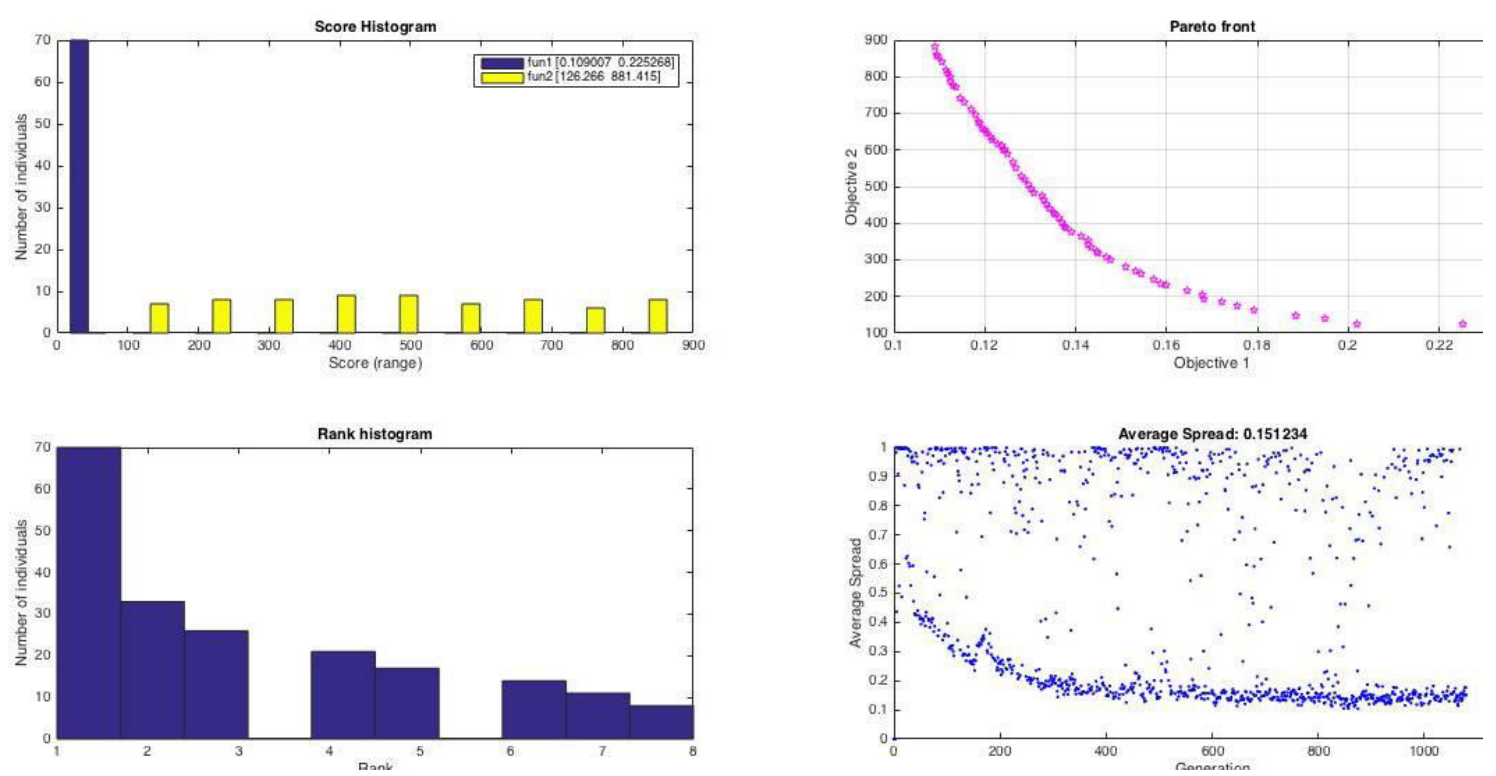

Table 4. Summary of Results of Single and Multi-objective GA optimization

\begin{tabular}{llllllll}
\hline Mix No. & $f^{\prime} c$ & $Q$ & $P C$ & $F A$ & $S F$ & $F$ & $C A$
\end{tabular}




\begin{tabular}{|c|c|c|c|c|c|c|c|c|}
\hline & (MPa) & (Coulomb) & $\left(\mathrm{kg} / \mathrm{m}^{3}\right)$ & $\left(\mathrm{kg} / \mathrm{m}^{3}\right)$ & $\left(\mathrm{kg} / \mathrm{m}^{3}\right)$ & $\left(\mathrm{kg} / \mathrm{m}^{3}\right)$ & $\left(\mathrm{kg} / \mathrm{m}^{3}\right)$ & $\left(\mathrm{kg} / \mathrm{m}^{3}\right)$ \\
\hline \multicolumn{9}{|c|}{ Single-Objective Problem using CSNN1 Model } \\
\hline 1 & 114 & - & 587 & 420 & 60 & 1,021 & 595 & 9.45 \\
\hline \multicolumn{9}{|c|}{ Single-Objective Problem using QPNN1 Model } \\
\hline 2 & - & $<0$ & 600 & 420 & 38.7 & 1,166 & 610.4 & 4.74 \\
\hline \multicolumn{9}{|c|}{ Multi-Objective Problem using CNN1 Model } \\
\hline 3 & 99.0 & 375 & 363 & 180 & 57 & 1,109 & 595 & 9.45 \\
\hline 4 & 102.5 & 881 & 328 & 238 & 36 & 1,110 & 595 & 9.45 \\
\hline 5 & 100.5 & 565 & 362 & 194 & 43 & 1,109 & 595 & 9.45 \\
\hline 6 & 97.4 & 269 & 362 & 180 & 60 & 1,110 & 595 & 4.91 \\
\hline 7 & 89.1 & 126 & 362 & 180 & 60 & 1,104 & 595 & 2.29 \\
\hline
\end{tabular}

E. Multi-objective GA optimization

After over 1,000 generations of the multi-objective GA optimization, pareto-front of 70 high performing mix proportions were determined (See Figure 9). In this final population, compressive strength of as high as 102.5 MPa was derived while charge passed was reduced to as low as 126 Coulomb. The pareto-front has an average compressive strength and charge passed of $99.1 \mathrm{MPa}$ and 483 Coulomb respectively. The standard deviations of the compressive strength and charge passed for the derived mixes are three and 224 respectively.

Five mix proportions were sorted based on their performance relative to high compressive strength and low charge passed. These high ranking mixes were summarized in Table 4. All five mixes exhibited high compressive strength over $60 \mathrm{MPa}$ with average w/b ratio of 0.30 . Chloride ion penetration resistance for the 5 samples were characterized by very low (100-1000 Coulombs) penetrability. The FA content varies from 30$40 \%$ while SF varies from $8-10 \%$ of the total binder weight.

It is indicative of the sensitivity of PC, FA, and SF content to the compressive strength and charge passed of the SCC. High SF content will not fully compensate the low pozzolanic reaction rate of FA thus it yields decrease in its compressive strength. This can be observed with Mix nos. 6 and 7, characterized by high SF content relative to the total binder weight. However high SF content improved the chloride ion penetration resistance (charge passed) and this was evidently present on the five mixes.

\section{CONCLUSION}

In this paper, the established good effects of high calcium FA and SF by previous studies as a binary and ternary cementitious blends at high volume replacement on the strength and durability properties of SCC was further investigated using two widely used biological inspired computing models namely the Artificial Neural Network (ANN) and Genetic Algorithm (GA). From these results it can be concluded that ANN and GA were able to utilize in developing a hybrid model use for prediction and optimization purposes. The hybrid model was able to explore material components of sustainable SCC particularly the FA and SF in effect to 28-day compressive strength and chloride ion penetration resistance, and optimize the proportion of material components that resulted to objective mix design of SCC considering strength and durability performance.
Furthermore, computational exploration of the influence was also carried out as to further understand the sensitivity of the two mineral admixtures to the strength-durability performance without the expense of experimental program. The model also provided the understanding of the viability of the two mineral admixtures (i.e. FA and SF) in developing sustainable SCC under strength-durability requirements.

\section{ACKNOWLEDGEMENT}

The Author wish to convey his deepest gratitude to the Technological Institute of the Philippines (T.I.P.) for supporting this academic endeavor through research grant fund and with the recommendation and support of the Department of Civil Engineering, College of Engineering and Architecture. In same way the author is also grateful to Engr. Nolan Concha of Department of Civil Engineering, FEU-Institute of Technology, Philippines and Dr. Watcharapong Wongkeo of Department of Physics and Materials Science, Chiang Mai University, Thailand for their invaluable inputs to this study.

\section{REFERENCES}

[1] H. Witkowski, "Sustainability of self-compacting concrete," Architecture Civil Engineering Environment, vol. 8, 2015.

[2] J. Thirumal and R. Harish, "Performance Study of SelfCompacting Concrete by Fly Ash and Silica Fume for Sustainability in Building Construction," in Key Engineering Materials, 2016, vol. 692, pp. 74-81: Trans Tech Publ.

[3] O. A. Mohamed and W. Al-Hawat, "Influence of Fly Ash and Basalt Fibers on Strength and Chloride Penetration Resistance of Self-Consolidating Concrete," in Materials Science Forum, 2016, vol. 866, pp. 3-8: Trans Tech Publ.

[4] W. Wongkeo, P. Thongsanitgarn, A. Ngamjarurojana, and A. Chaipanich, "Compressive strength and chloride resistance of self-compacting concrete containing high level fly ash and silica fume," Materials \& Design, vol. 64, pp. 261-269, 2014.

[5] R. Venkatakrishnaiah and G. Sakthivel, "Bulk utilization of flyash in self compacting concrete," KSCE Journal of Civil Engineering, vol. 19, no. 7, p. 2116, 2015.

[6] J. G. Jawahar, C. Sashidhar, I. R. Reddy, and J. A. Peter, "Design of cost-effective M 25 grade of self compacting concrete," Materials \& Design, vol. 49, pp. 687-692, 2013.

[7] J. G. Jawahar, C. Sashidhar, I. R. Reddy, and J. A. Peter, "Micro and macrolevel properties of fly ash blended self compacting concrete," Materials \& Design, vol. 46, pp. 696-705, 2013. 
[8] M. Sonebi and P. Bartos, "Filling ability and plastic settlement of self-compacting concrete," Materials and structures, vol. 35, no. 8, pp. 462-469, 2002.

[9] R. Khurana and R. Saccone, "Fly ash in self-compacting concrete," Special Publication, vol. 199, pp. 259-274, 2001.

[10] K. H. Khayat, J. Bickley, and M. Lessard, "Performance of self-consolidating concrete for casting basement and foundation walls," Materials Journal, vol. 97, no. 3, pp. 374-380, 2000.

[11] M. Gesoğlu, E. Güneyisi, and E. Özbay, "Properties of self-compacting concretes made with binary, ternary, and quaternary cementitious blends of fly ash, blast furnace slag, and silica fume," Construction and Building Materials, vol. 23, no. 5, pp. 1847-1854, 2009.

[12] N. C. Concha, "Rheological Optimization of Self Compacting Concrete with Sodium Lignosulfate Based Accelerant Using Hybrid Neural Network-Genetic Algorithm," in Materials Science Forum, 2016, vol. 866, pp. 9-13: Trans Tech Publ.

[13] N. C. Concha and E. P. Dadios, "Optimization of the rheological properties of self compacting concrete using neural network and genetic algorithm," in Humanoid, Nanotechnology, Information Technology, Communication and Control, Environment and Management (HNICEM), 2015 International Conference on, 2015, pp. 1-6: IEEE.

[14] H. Adeli and S.-L. Hung, Machine learning: neural networks, genetic algorithms, and fuzzy systems. John Wiley \& Sons, Inc., 1994.

[15] M. Affenzeller, S. Wagner, S. Winkler, and A. Beham, Genetic algorithms and genetic programming: modern concepts and practical applications. Crc Press, 2009.

[16] R. L. Haupt and S. E. Haupt, Practical genetic algorithms. John Wiley \& Sons, 2004.

[17] B. STANDARD, "Testing hardened concrete," 2009.

[18] P. Chopra, R. K. Sharma, and M. Kumar, "Prediction of Compressive Strength of Concrete Using Artificial
Neural Network and Genetic Programming," Advances in Materials Science and Engineering, vol. 2016, 2016.

[19] P. Asteris, K. Kolovos, M. Douvika, and K. Roinos, "Prediction of self-compacting concrete strength using artificial neural networks," European Journal of Environmental and Civil Engineering, vol. 20, no. sup1, pp. s102-s122, 2016.

[20] M. Taner, "Neural networks and computation of neural network weights and biases by the generalized delta rule and back-propagation of errors," Rock solid images, 1995.

[21] Q.-J. Zhang, K. C. Gupta, and V. K. Devabhaktuni, "Artificial neural networks for RF and microwave design-from theory to practice," IEEE transactions on microwave theory and techniques, vol. 51, no. 4, pp. 1339-1350, 2003.

[22] D. E. Goldberg, "Genetic algorithms in search, optimization and machine learning 'addison-wesley, 1989," Reading, MA, 1989.

[23] C.-H. Lim, Y.-S. Yoon, and J.-H. Kim, "Genetic algorithm in mix proportioning of high-performance concrete," Cement and Concrete Research, vol. 34, no. 3, pp. 409-420, 2004.

[24] J. E. Baker, "Reducing bias and inefficiency in the selection algorithm," in Proceedings of the second international conference on genetic algorithms, 1987, pp. 14-21.

[25] S. Marsili Libelli and P. Alba, "Adaptive mutation in genetic algorithms," Soft Computing-A Fusion of Foundations, Methodologies and Applications, vol. 4, no. 2, pp. 76-80, 2000.

[26] W. Chalee, M. Teekavanit, K. Kiattikomol, A. Siripanichgorn, and C. Jaturapitakkul, "Effect of W/C ratio on covering depth of fly ash concrete in marine environment," Construction and Building Materials, vol. 21, no. 5, pp. 965-971, 2007. 